\title{
GENERATION OF AXISYMMETRIC MAGNETOSONIC SHOCK WAVES: APPLICATIONS TO STATIC EQUILIBRIA AND ACCRETION DISKS
}

\author{
Manuel Núñez \\ Depto. Análisis Matemático \\ Universidad de Valladolid \\ 47011 Valladolid, Spain \\ e-mail:mnjmhd@am.uva.es
}

\begin{abstract}
Magnetosonic waves traveling in a MHD equilibrium may evolve into shocks. We develop a criterion for the creation of fast shocks in the equatorial plane of axisymmetric equilibria and analyze the influence of the most important parameters. The results are applied to Grad-Shafranov equilibria and accretion disks.
\end{abstract}

PACS numbers: 02.30.Jr, 52.35.Bj, 52.35.Tc

Keywords: Shock waves, fast magnetosonic waves, MHD equilibria, accretion disks

\section{Introduction}

This paper deals with the creation of magnetosonic shock waves in axisymmetric MHD equilibria. While the theoretical results hold for all cases, the practical application of them requires a knowledge of the particular situation we are 
dealing with. We will concentrate on two important instances: static equilibria described by the Grad-Shafranov equation, which play such a significant part in the analysis of stability of confined plasmas; and accretion rings near astrophysical objects ranging from planets to stars to black holes. In the last instance the full relativistic approach would be necessary, but as soon as we leave the immediate vicinity of the object the Newtonian equations are extremely precise. Since each of these topics has its own rationale, we introduce them in turn.

Perhaps the most successful endeavor in classical Magnetohydrodynamics was the proof of the existence of one and two-dimensional static equilibria, as well as the analysis of their linear stability. Among the many references we may select the classical review [1], and the excellent modern exposition [2]. One of the aims of this theory is to identify perturbation frequencies which lead to exponential growth and therefore show the instability of the equilibrium. As befits a linear theory, this does not depend on the initial size of the perturbation, provided it is small enough for the linearized MHD equations to be considered valid. Nonlinear stability depends more heavily on computational algorithms, but there is an important instance where we can go a long way by analytical methods. This concerns the propagation of MHD waves into an unperturbed state, a subject forming part of the study of nonlinear hyperbolic systems [3,4]. Both sound and MHD waves have been studied in simple special configurations from a long time ago [5-8], but never including the case of axisymmetric equilibria.

Accretion disks form one of the most conspicuous astrophysical structures, present e.g. in galactic nuclei, young and dwarf stars, black holes, binary systems and wherever jets are observed. Among the plethora of general monographs and review articles dealing with this topic, [9-11] may be commended by their breadth and clarity. Although perhaps the main object of theoretical interest today is the presence and consequences of the magnetorotational instability [12-14], the existence of shocks is also relevant [15]. In fact, one of its effects is plasma heating, which remains one of the most relevant topics in the study of accretion disks [16].

There are several analytic approaches to the problem $[17,18]$ of shock formation, but all lead to a differential equation satisfied along the rays by the jump 
of the time derivative of the solution at the wavefront separating the perturbed and the unperturbed states. This equation is of Riccati type and may lead to a blow up of the solution in a finite time, a fact which is interpreted as the formation of a discontinuity in both velocity and magnetic field consistent with the formation of a shock wave. A MHD shock wave yields several undesirable effects such as the creation of surface currents in the plasma, which does not bode well for the preservation of the equilibrium; and shock waves in accretion diks are equally disrupting. Being a nonlinear feature, the size of the initial perturbation is a key parameter governing the future evolution of the system. While most classical papers assume that in the state where the wave propagates all the quantities are constant, this simplifying hypothesis does not hold for nontrivial static equilibria, both in the static case an in accretion disks. Fortunately the general case is also included in modern treatises on nonlinear waves, such as $[19,20]$.

Let us end the introduction by admitting some weaknesses. Since the only comprehensive theory of nonlinear waves and shocks involve quasilinear hyperbolic systems, we must use the ideal MHD system, thus ignoring both resistive and turbulent dissipation. Also the geometry is constrained, not only because of axisymmetry but also because of several North-South symmetries we will impose; and finally, to descend to precise predictions, we must consider certain simple configurations of the main quantities: low beta and poloidal field for static settings, self-similar behavior for accretion disks. On the positive side, we will obtain rigorous criteria guaranteeing that a certain perturbation located at a fixed radius will eventually evolve into a fast magnetosonic shock, and we will be able to pinpoint the exact location where this will occur.

\section{General results}

Since the main results on nonlinear waves propagating into an equilibrium are not so well known, we will recall them briefly. Let

$$
\frac{\partial \mathbf{u}}{\partial t}+\sum_{j=1}^{3} A_{j}(t, \mathbf{x}, \mathbf{u}) \frac{\partial \mathbf{u}}{\partial x_{j}}+\mathbf{C}(t, \mathbf{x}, \mathbf{u})=\mathbf{0}
$$


be a quasilinear hyperbolic system, where all functions are assumed smooth enough. Let $\mathbf{u}_{0}$ be a known stationary solution of the system (1). Let $\tau(t, \mathbf{x})$ be a solution of the eikonal equation

$$
\operatorname{det}\left(\sum_{j=1}^{3} A_{j}\left(t, \mathbf{x}, \mathbf{u}_{0}\right) \frac{\partial \tau}{\partial x_{j}}+\frac{\partial \tau}{\partial t} I\right)=0 .
$$

Assume that $\partial \tau / \partial t$ is an eigenvalue of order one of the matrix

$$
\sum_{j=1}^{3} A_{j}\left(t, \mathbf{x}, \mathbf{u}_{0}\right) \frac{\partial \tau}{\partial x_{j}}
$$

and let $\mathbf{R}$ be a right eigenvector of this matrix, $\mathbf{L}$ a left eigenvector. Let $\Omega(t): \tau(t, \mathbf{x})=$ const., be a level surface of $\tau$ (a characteristic surface) separating two states, one of which is precisely $\mathbf{u}_{0}$. Equation (2) corresponds to the characteristic surfaces of the system. Assume that the variable solution propagates into the state $\mathbf{u}=\mathbf{u}_{0}$, where the normal vector to $\Omega$ points. This means that

$$
\frac{\partial \tau}{\partial t}<0, \quad \mathbf{n}=\frac{\nabla \tau}{|\nabla \tau|}
$$

and the velocity of $\Omega$ is

$$
c=-\frac{\partial \tau / \partial t}{|\nabla \tau|} .
$$

We also assume that $\mathbf{u}$ is continuous at both sides of $\Omega$, but perhaps not its derivatives. In fact, let [ ] denote the jump at $\Omega$, i.e. the magnitude at the positive side of $\Omega, \mathbf{u}=\mathbf{u}_{0}$, minus the one on the negative side. Then

$$
\left[\frac{\partial \mathbf{u}}{\partial t}\right]=w_{0} \mathbf{R},
$$

for a scalar $w_{0}(t, \mathbf{x})$ whose evolution along the rays satisfies a certain Riccati equation which we will detail later. Eigenvectors are determined up to a multiplicative constant, so that $w_{0}$ depends on our particular choosing; nevertheless, the left hand side of (6) is independent of it, as well as the time when shock waves occur. Rays are the bicharacteristic curves of system (1), i.e. solutions of the following equations: if $c=c(\mathbf{n}, \mathbf{x}, t)$ is the velocity (5), a ray $t \rightarrow \mathbf{x}(t)$ 
satisfies

$$
\begin{array}{r}
\frac{d x_{i}}{d t}=c n_{i}+\left(\delta_{i j}-n_{i} n_{j}\right) \frac{\partial c}{\partial n_{j}}, \\
\frac{d n_{i}}{d t}=\left(n_{i} n_{j}-\delta_{i j}\right) \frac{\partial c}{\partial x_{j}} .
\end{array}
$$

It is known that in general rays are not orthogonal to wavefronts: this is guaranteed only if the speed of propagation $c$ does not depends on $\mathbf{n}$. Since all the quantities are evaluated at $\mathbf{u}=\mathbf{u}_{0}$, this is an ordinary differential equation in $(t, \mathbf{x})[5]$. For any matrix or vector $B(t, \mathbf{x}, \mathbf{u})$ we use the following notation:

$$
\left(\nabla_{u} B\right) \cdot \mathbf{R}=\sum_{i} R_{i} \frac{\partial B}{\partial u_{i}},
$$

all of them evaluated at $\mathbf{u}_{0}$. Let

$$
\begin{array}{r}
q_{0}=\mathbf{L} \cdot\left(\sum_{j=1}^{3} \frac{n_{j}}{c}\left(\nabla_{u} A_{j}\right) \cdot \mathbf{R}\right) \cdot \mathbf{R}, \\
p_{0}=\mathbf{L} \cdot\left(\sum_{j=1}^{3} A_{j} \frac{\partial \mathbf{R}}{\partial x_{j}}+\sum_{j=1}^{3}\left(\nabla_{u} A_{j} \cdot \mathbf{R}\right) \cdot\left(\frac{\partial \mathbf{u}_{0}}{\partial x_{j}}\right)\right)+\mathbf{L} \cdot\left(\nabla_{u} \mathbf{C}\right) \cdot \mathbf{R},
\end{array}
$$

The expression in (9) depends only on the values of the vectors $\mathbf{L}$ and $\mathbf{R}$ at a single point, which means that once chosen a fixed normal vector $\mathbf{n}, \mathbf{L}$ and $\mathbf{R}$ are left and right eigenvectors of $A_{n}=\sum n_{j} A_{j}$. Then

$$
q_{0}=\mathbf{L} \cdot\left(\frac{1}{c}\left(\nabla_{u} A_{n}\right) \cdot \mathbf{R}\right) \cdot \mathbf{R} .
$$

This value depends only on the normal, and not on the geometry of the wavefront. The same may be said of the product $\mathbf{L} \cdot \mathbf{R}$. Things are different for (10); this term involves derivatives of the quantities which must be found along a wavefront, so we need some information on the local geometry of the surface. If we parametrize the surface $\Omega$ in the form $x_{i}=x_{i}\left(y_{1}, y_{2}, t\right)$, and $\left(g_{\alpha \beta}\right)$ is the metric tensor of the surface, we have

$$
\begin{array}{r}
p_{0}=\mathbf{L} \cdot\left(A_{n} \frac{\partial \mathbf{R}}{\partial n}+\sum_{j, \alpha, \beta} A_{j} g^{\alpha \beta} \frac{\partial x_{j}}{\partial y_{\beta}} \frac{\partial \mathbf{R}}{\partial y_{\alpha}}\right) \\
+\mathbf{L} \cdot\left(\left[\nabla_{u} A_{n} \cdot \mathbf{R}\right] \frac{\partial \mathbf{u}_{0}}{\partial n}+\sum_{j, \alpha, \beta}\left[\nabla_{u} A_{j} \cdot \mathbf{R}\right] g^{\alpha \beta} \frac{\partial x_{j}}{\partial y_{\beta}} \frac{\partial \mathbf{u}_{0}}{\partial y_{\alpha}}\right)+\mathbf{L} \cdot\left(\nabla_{u} \mathbf{C}\right) \cdot \mathbf{R} .
\end{array}
$$


The main result is as follows: let $t \rightarrow \mathbf{x}(t)$ represent a ray associated to the phase $\tau, w(t)=w_{0}(t, \mathbf{x}(t)), p=(\mathbf{L} \cdot \mathbf{R})^{-1} p_{0}, q=(\mathbf{L} \cdot \mathbf{R})^{-1} q_{0}$. Then $w$ satisfies

$$
\frac{d w}{d t}+p w+q w^{2}=0
$$

Since this Riccati equation lacks an independent term, it may be immediately reduced to a linear one:

$$
\frac{d}{d t}\left(\frac{1}{w}\right)-\frac{p}{w}-q=0
$$

whose solution is

$$
\begin{array}{r}
\frac{1}{w(t)}=\frac{1}{w\left(t_{0}\right)} \exp \left(\int_{t_{0}}^{t} p(s) d s\right) \\
+\exp \left(\int_{t_{0}}^{t} p(s) d s\right) \int_{t_{0}}^{t} \exp \left(-\int_{t_{0}}^{s} p(r) d r\right) q(s) d s .
\end{array}
$$

Therefore, if there exists $t_{1}$ such that

$$
\frac{1}{w\left(t_{0}\right)}=-\int_{t_{0}}^{t_{1}} \exp \left(-\int_{0}^{s} p(r) d r\right) q(s) d s,
$$

then $w(t)$ tends to $\infty$ when $t \rightarrow t_{1}$, which, provided that $\mathbf{R}$ does not tend to zero, means that the jump of the differential of $\mathbf{u}$ tends to infinity. Hence $\mathbf{u}$ undergoes a jump and becomes discontinuous, i.e. a shock appears. The interval of time where the integral is evaluated depends on the problem; it may be limited by the physical characteristics of the process under study (as in our case, by the dimensions of the device or the accretion disk where the equilibrium exists) or by the possible formation of caustics and loss of regularity of the wavefront. On the other hand, if $w\left(t_{0}\right)>0$ and the integral in (16) tends to $\infty$, then $w(t) \rightarrow 0$ and the wavefront becomes a mild discontinuity.

\section{Propagation in axisymmetric equilibria}

Obviously our first aim is to state the equations of ideal MHD in cylindrical coordinates $(z, r, \phi)$. The main magnitudes are velocity, magnetic field and two of three thermodynamic quantities: density $\rho$, entropy $S$ and pressure $P$, related by a state equation. The entropy uncouples from the rest and may be ignored. We will follow ( [21], p. 16) (notice that there are a few misprints in this text) 
and use the density as primary variable. In order to simplify the result, will assume from the beginning axisymmetry $(\partial / \partial \phi=0)$. Let us denote the velocity and magnetic field by

$$
\begin{array}{r}
\mathbf{v}=v_{z} \hat{z}+v_{r} \hat{r}+v_{\phi} \hat{\phi} \\
\mathbf{B}=B_{z} \hat{z}+B_{r} \hat{r}+B_{\phi} \hat{\phi} .
\end{array}
$$

Let $I_{7}$ denote the $7 \times 7$ identity matrix. The main equations are

$$
\begin{aligned}
& \frac{\partial}{\partial t}\left[\begin{array}{c}
v_{z} \\
v_{r} \\
v_{\phi} \\
B_{z} \\
B_{r} \\
B_{\phi} \\
\rho
\end{array}\right]+\left(v_{z} I_{7}+\left[\begin{array}{ccccccc}
0 & 0 & 0 & 0 & B_{r} / \rho & B_{\phi} / \rho & P_{\rho} / \rho \\
0 & 0 & 0 & 0 & -B_{z} / \rho & 0 & 0 \\
0 & 0 & 0 & 0 & 0 & -B_{z} / \rho & 0 \\
0 & 0 & 0 & 0 & 0 & 0 & 0 \\
B_{r} & -B_{z} & 0 & 0 & 0 & 0 & 0 \\
B_{\phi} & 0 & -B_{z} & 0 & 0 & 0 & 0 \\
\rho & 0 & 0 & 0 & 0 & 0 & 0
\end{array}\right]\right) \frac{\partial}{\partial z}\left[\begin{array}{c}
v_{z} \\
v_{r} \\
v_{\phi} \\
B_{z} \\
B_{r} \\
B_{\phi} \\
\rho
\end{array}\right] \\
& +\left(v_{r} I_{7}+\left[\begin{array}{ccccccc}
0 & 0 & 0 & -B_{r} / \rho & 0 & 0 & 0 \\
0 & 0 & 0 & B_{z} / \rho & 0 & B_{\phi} / \rho & P_{\rho} / \rho \\
0 & 0 & 0 & 0 & 0 & -B_{r} / \rho & 0 \\
-B_{r} & B_{z} & 0 & 0 & 0 & 0 & 0 \\
0 & 0 & 0 & 0 & 0 & 0 & 0 \\
0 & B_{\phi} & -B_{r} & 0 & 0 & 0 & 0 \\
0 & \rho & 0 & 0 & 0 & 0 & 0
\end{array}\right]\right) \frac{\partial}{\partial r}\left[\begin{array}{c}
v_{z} \\
v_{r} \\
v_{\phi} \\
B_{z} \\
B_{r} \\
B_{\phi} \\
\rho
\end{array}\right] \\
& +\left[\begin{array}{c}
0 \\
-v_{\phi}^{2} / r+B_{\phi}^{2} / \rho r \\
v_{r} v_{\phi} / r-B_{r} B_{\phi} / \rho r \\
B_{z} v_{r} / r \\
B_{r} v_{r} / r \\
\rho v_{\phi} / r \\
\rho v_{r} / r
\end{array}\right]+\left[\begin{array}{c}
\frac{G M}{\left(r^{2}+z^{2}\right)^{3 / 2}} z \\
\frac{G M}{\left(r^{2}+z^{2}\right)^{3 / 2}} r \\
0 \\
0 \\
0 \\
0 \\
0
\end{array}\right]=\mathbf{0 .}
\end{aligned}
$$

We will abbreviate this to

$$
\frac{\partial \mathbf{u}}{\partial t}+A_{z} \frac{\partial \mathbf{u}}{\partial z}+A_{r} \frac{\partial \mathbf{u}}{\partial r}+\mathbf{C}=\mathbf{0}
$$


Without axisymmetry a further matrix $A_{\phi}$ would appear. Although later we will write it formally its specific value will not be necessary. Also the last vector, representing the gravitational force ( $G$ is the gravitational constant and $M$ the mass of the central object) applies only to accretions disks, and disappears for laboratory plasmas.

We will set a number of conditions on the velocity and the field which will be different in each case, but all have in common that the propagation velocity $c$ is not only axisymmetric, but even with respect to the variable $z$. Thus, if the initial value $\tau(0)$ is axisymmetric, wavefronts will intersect the equator $\Pi: z=0$ as circumferences and their normal vectors will be radial. We will limit our study to fast magnetosonic shock waves occurring first in the plane $\Pi$. The choosing of fast waves is logical: because they have the largest velocity, they will be the ones to extend into an untouched equilibrium state; moreover, they are the only ones which may develop across the equilibrium magnetic field, which will be needed in our geometry. While one could imagine wavefront configurations creating shocks above and below $\Pi$, usually compression is maximized at the plane and one expects shocks beginning at the plane of symmetry. Thus the normal matrix $A_{n}$ coincides with $A_{r}$. The eigenvalue associated to the fast magnetosonic wave is given by $v_{n} \pm \mu$, where $v_{n}$ is the velocity of the fluid normal to the wavefront, and

$$
2 \mu^{2}=c^{2}+\sqrt{c^{4}-\frac{4 B_{r}^{2} P_{\rho}}{\rho}},
$$

where $c$ is the total velocity, sum of the sound and Alfvén ones:

$$
c^{2}=P_{\rho}+\frac{B^{2}}{\rho} .
$$

Provided $\mu^{2} \neq B_{r}^{2} / \rho$ (which never happens unless $P_{\rho}=B_{z}=B_{\phi}=0$ ), the right and left eigenvectors turn out to be

$$
\begin{aligned}
\mathbf{R} & =\left(-\frac{B_{z} B_{r}}{\rho \mu}, \mu-\frac{B_{r}^{2}}{\rho \mu},-\frac{B_{r} B_{\phi}}{\rho \mu}, B_{z}, 0, B_{\phi}, \frac{\rho}{\mu}\left(\mu-\frac{B_{r}^{2}}{\mu}\right)\right), \\
\mathbf{L} & =\left(-\frac{B_{z} B_{r}}{\mu}, \rho\left(\mu-\frac{B_{r}^{2}}{\rho \mu}\right),-\frac{B_{r} B_{\phi}}{\mu}, B_{z}, 0, B_{\phi}, \frac{P_{\rho}}{\mu}\left(\mu-\frac{B_{r}^{2}}{\mu}\right)\right),
\end{aligned}
$$

up to multiplication by a real constant. We defer the calculations in this section to the appendix; for typographical convenience we write vectors as rows instead 
of columns. We will see that in all our examples, in the plane $\Pi$ we have $B_{r}=0$; thus those vectors simplify to

$$
\begin{aligned}
& \mathbf{R}=\left(0, c, 0, B_{z}, 0, B_{\phi}, \rho\right), \\
& \mathbf{L}=\left(0, \rho c, 0, B_{z}, 0, B_{\phi}, P_{\rho}\right) .
\end{aligned}
$$

To find $\partial \mathbf{R} / \partial r$ at $\Pi$ we may use (25), but for $\partial \mathbf{R} / \partial z$ we need the full formula (23). Anyway, at $\Pi$,

$$
\mathbf{L} \cdot \mathbf{R}=\rho c^{2}+B_{z}^{2}+B_{\phi}^{2}+\rho P_{\rho}=2 \rho c^{2} .
$$

We need now to evaluate all the terms in the expressions of $q_{0}$ and $p_{0}$. Relegating again a scheme of the calculations to the Appendix, one finds in all cases

$$
q_{0}=2 \rho c^{2}+B^{2}+\rho^{2} P_{\rho \rho}
$$

and

$$
\begin{aligned}
\mathbf{L} \cdot A_{r} \cdot \frac{\partial \mathbf{R}}{\partial r} & =c \frac{\partial}{\partial r}\left(P+\frac{B^{2}}{2}\right)+\rho c^{2} \frac{\partial c}{\partial r}, \\
\mathbf{L} \cdot\left(\nabla_{u} \mathbf{C}\right) \cdot \mathbf{R} & =\frac{\rho c^{3}}{r} .
\end{aligned}
$$

The state $\mathbf{u}_{0}$ will have a different form in each case, but they will always satisfy at the plane $\Pi$

$$
\mathbf{L} \cdot\left(\nabla_{u} A_{r} \cdot \mathbf{R}\right) \cdot \frac{\partial \mathbf{u}_{0}}{\partial r}=c\left(\frac{1}{2} \frac{\partial B^{2}}{\partial r}+\rho P_{\rho \rho} \frac{\partial \rho}{\partial r}\right) .
$$

It remains to find the terms where tangential derivatives to the wavefront occur. We know nothing about the wavefront surface other than it is axisymmetric and $z$-symmetric. We may parametrize it by its section by the plane $\phi=0$, a curve $z \rightarrow r(z)$, which satisfies $r(z)=r(-z)$, so that $r^{\prime}(0)=0$. The surface will be $\mathbf{x}(z, \phi)=(r(z) \cos \phi, r(z) \sin \phi, z)$. Thus

$$
g^{z z}=\frac{1}{1+r^{\prime}(z)^{2}}, \quad g^{\phi \phi}=\frac{1}{r(z)^{2}}, \quad g^{z \phi}=0 .
$$

Thus, for any axisymmetric vector field $\mathbf{F}$ and matrices $\mathcal{A}_{j}$,

$$
\sum_{j, \alpha, \beta} \mathcal{A}_{j} g^{\alpha \beta} \frac{\partial x_{j}}{\partial y_{\beta}} \frac{\partial \mathbf{F}}{\partial y_{\alpha}}=\mathcal{A}_{z} \frac{\partial \mathbf{F}}{\partial z}
$$


This is applied to the vectors and matrices $\mathbf{F}=\mathbf{R}$ with $\mathcal{A}_{j}=A_{j}$, as well as $\mathbf{F}=\mathbf{u}_{0}$ with $\mathcal{A}_{j}=\nabla_{u} A_{j} \cdot \mathbf{R}$. Now it is easy to see (shown in the appendix) that the second term in the expression of $p_{0}$ in (12) becomes

$$
\mathbf{L} \cdot\left(\sum_{j, \alpha, \beta} A_{j} g^{\alpha \beta} \frac{\partial x_{j}}{\partial y_{\beta}} \frac{\partial \mathbf{R}}{\partial y_{\alpha}}\right)=\mathbf{L} \cdot A_{z} \frac{\partial \mathbf{R}}{\partial z}=-\frac{B_{z} P_{\rho}}{c} \frac{\partial B_{r}}{\partial z} .
$$

Finally

$$
\mathbf{L} \cdot\left(\sum_{j, \alpha, \beta}\left[\nabla_{u} A_{j} \cdot \mathbf{R}\right] g^{\alpha \beta} \frac{\partial x_{j}}{\partial y_{\beta}} \frac{\partial \mathbf{u}_{0}}{\partial y_{\alpha}}\right)=\mathbf{L} \cdot\left[\nabla_{u} A_{z} \cdot \mathbf{R}\right] \frac{\partial \mathbf{u}_{0}}{\partial z}=-B_{z} c \frac{\partial B_{r}}{\partial z} .
$$

Adding (29), (30), (31), (34) and (35), we obtain

$$
\begin{gathered}
p_{0}=c \frac{\partial}{\partial r}\left(P+\frac{B^{2}}{2}\right)+\rho c^{2} \frac{\partial c}{\partial r}+c\left(\frac{1}{2} \frac{\partial B^{2}}{\partial r}+\rho P_{\rho \rho} \frac{\partial \rho}{\partial r}\right) \\
+\frac{\rho c^{3}}{r}-\frac{B_{z}\left(P_{\rho}+c^{2}\right)}{c} \frac{\partial B_{r}}{\partial z}=\frac{1}{r} \frac{\partial}{\partial r}\left(r c^{3} \rho\right)-\frac{B_{z}\left(P_{\rho}+c^{2}\right)}{c} \frac{\partial B_{r}}{\partial z} .
\end{gathered}
$$

Thus

$$
\begin{array}{r}
p=\frac{p_{0}}{2 \rho c^{2}}=c \frac{\partial}{\partial r} \ln \left(\sqrt{\rho c^{3} r}\right)-\frac{B_{z}\left(P_{\rho}+c^{2}\right)}{2 \rho c^{3}} \frac{\partial B_{r}}{\partial z}, \\
q=\frac{2 \rho c^{2}+B^{2}+\rho^{2} P_{\rho \rho}}{2 \rho c^{2}} .
\end{array}
$$

Our next step is to find the displacement along the rays, which as we know are radial. Equations (7) simplify because by axisymmetry $c$ depends only on $r$ at $\Pi$; thus

$$
\begin{aligned}
\frac{d \mathbf{n}}{d t}=\left(n^{2}-1\right) \frac{d c}{d r} \hat{r} & =\mathbf{0}, \\
\frac{d \mathbf{x}}{d t} & =c \hat{r},
\end{aligned}
$$

which means that as expected the rays are traveled at speed $c$. Thus $d / d t=$ $c d / d r$. Let us write (13) in terms of the parameter $r$ :

$$
\frac{d w}{d r}+\left[\frac{\partial}{\partial r} \ln \sqrt{\rho c^{3} r}-\frac{B_{z} \rho\left(P_{\rho}+c^{2}\right)}{2 \rho^{2} c^{4}} \frac{\partial B_{r}}{\partial z}\right] w+\frac{1}{c}\left[\frac{2 \rho c^{2}+B^{2}+\rho^{2} P_{\rho \rho}}{2 \rho c^{2}}\right] w^{2}=0 .
$$

The first factor in the exponential of the integral of $p$ (see (15) may be found easily:

$$
\exp \left(-\int_{r_{0}}^{r} \frac{\partial}{\partial s} \ln \sqrt{\rho c^{3} s} d s\right)=\sqrt{\frac{\rho_{0} c_{0}^{3} r_{0}}{\rho c^{3} r}}
$$


Thus equation (16) may be written in terms of the radii

$$
=\int_{r_{0}}^{r_{1}}\left(\frac{\rho_{0} c_{0}^{3} r_{0}}{\rho c^{3} r}\right)^{1 / 2}\left(1+\frac{B^{2}+\rho^{2} P_{\rho \rho}}{2 \rho c^{2}}\right) \exp \left(\int_{r_{0}}^{s} \frac{B_{z} \rho\left(P_{\rho}+c^{2}\right)}{2 \rho^{2} c^{4}} \frac{\partial B_{r}}{\partial z} d s\right) \frac{1}{c} d r,
$$

where the subindex 0 means that the quantities are taken at the point $r_{0}$, and

$$
\left[\frac{\partial \mathbf{u}}{\partial t}\right]_{r=r_{0}}=w\left(r_{0}\right) \mathbf{R}\left(r_{0}\right) \text {. }
$$

Let us consider now equation (43) to see the terms contributing to the blow up of the solution. There is little to say in general for the term $q$ in (16) given by (41), other than the obvious bound

$$
q=\left(1+\frac{B^{2}+\rho^{2} P_{\rho \rho}}{2 \rho c^{2}}\right) \frac{1}{c} \geq \frac{1}{c} .
$$

For polytropic plasmas $P=A \rho^{\gamma}$ with $\gamma \leq 2$, we also have the upper bound

$$
q \leq \frac{3}{2 c},
$$

thus we cannot expect great effects from this term. The remaining factor is the only one where the magnetic field configuration outside the plane $\Pi$ plays any role. Since the focusing of compressive waves in the central plane may be an important source of shocks, we will analyze this term carefully. First we relate it to the curvature of poloidal field lines. Let us take a parametrization of this field line as $\xi \rightarrow(r(\xi), z(\xi))$, where $\dot{r}=B_{r}, \dot{z}=B_{z}$. As we know, the curvature may be written as

$$
\kappa=\frac{\dot{r} \ddot{z}-\ddot{r} \dot{z}}{\left(\dot{r}^{2}+\dot{z}^{2}\right)^{3 / 2}}=\frac{B_{r} \dot{B}_{z}-\dot{B}_{r} B_{z}}{\left(B_{z}^{2}+B_{r}^{2}\right)^{3 / 2}} .
$$

At the plane $\Pi, B_{r}=0$, so that $\kappa=-\dot{B}_{r} / B_{z}^{2}$. Thus

$$
B_{z} \frac{\partial B_{r}}{\partial z}=\frac{d z}{d \xi} \frac{\partial B_{r}}{\partial z}=\dot{B}_{r}=-B_{z}^{2} \kappa
$$

Thus the remaining factor in the integral in (43) may be written as

$$
\exp \left(\int_{r_{0}}^{r} \frac{B_{z} \rho\left(P_{\rho}+c^{2}\right)}{2 \rho^{2} c^{4}} \frac{\partial B_{r}}{\partial z} d s\right)=\exp \left(-\int_{r_{0}}^{r} \frac{B_{z}^{2} \rho\left(P_{\rho}+c^{2}\right)}{2 \rho^{2} c^{4}} \kappa d s\right) .
$$


Hence the exponent is positive whenever $\kappa<0$, negative for $\kappa>0$. The first case correspond to field lines concave with respect to $r=0$, as occurs in the left hand side of the magnetic axis, provided the equilibrium configuration possesses such an axis; and the second to convex poloidal lines, as to the right of the magnetic axis. Since the exponential grows very rapidly with the exponent, concavity in the direction of the positive $r$-axis is an important factor in the formation of shock waves. This is very intuitive: this geometry tends to push the fluid towards the central plane, thus creating a compressive wave that may evolve into a shock. To quantify all the terms ocurring in (43), we must descend to particular cases.

\section{Shock waves in specific axisymmetric equilib- ria}

\subsection{Static equilibria}

The initial state for a static axisymmetric plasma has the form

$$
\mathbf{u}_{0}=\left(0,0,0, B_{z}, B_{r}, B_{\phi}, \rho\right)
$$

As for the north-south symmetry, if we wish to preserve the classical picture of magnetic field lines coiling around tori, we must demand $B_{z}$ even, $B_{r}$ odd, $B_{\phi}$ even, $\rho$ even. This implies that at $z=0$,

$$
\frac{\partial B_{z}}{\partial z}=0, \quad B_{r}=0, \quad \frac{\partial B_{\phi}}{\partial z}=0 .
$$

Thus, in the plane $\Pi$ (see appendix),

$$
\frac{\partial \mathbf{u}_{0}}{\partial r}=\left(0,0,0, \frac{\partial B_{z}}{\partial r}, 0, \frac{\partial B_{\phi}}{\partial r}, \frac{\partial \rho}{\partial r}\right),
$$

and

$$
\frac{\partial \mathbf{u}_{0}}{\partial z}=\left(0,0,0,0, \frac{\partial B_{r}}{\partial z}, 0,0\right)
$$

All possible static axisymmetric equilibria may be found by solving the GradShafranov equation. Among them, the family of specific equilibria found by 
Solov'ev is well known [2]. Even these analytic examples become impossibly complex when applied to equation (43) with full generality of parameters. Thus we take simplicity a step further by assuming a plasma of very low beta, so that the pressure may be taken as constant and the speed of sound as negligible when compared to the Alfvén speed. Also we want to keep the main characteristic of tokamaks, the presence of a magnetic axis where the magnetic field is toroidal. Thus we choose the Solov'ev parameters so as to take the flux function

$$
\psi(r, z)=\left(r^{2}-r_{m}^{2}\right)^{2}-4 r^{2} z^{2},
$$

whose magnetic axis is located at $r=r_{m}, z=0$, and take $P=P_{0}, I=0$ (purely poloidal field). In these conditions, at the plane $\Pi$,

$$
\begin{gathered}
B_{r}=-8 r^{2} z, \quad \frac{\partial B_{r}}{\partial z}=-8 r^{2}, \quad B_{z}=4\left(r^{2}-r_{m}^{2}\right), \\
c=\frac{4}{\sqrt{\rho}}\left|r^{2}-r_{m}^{2}\right|, \quad \rho c^{2}=B^{2}=16\left(r^{2}-r_{m}^{2}\right)^{2} .
\end{gathered}
$$

Let us study the factors in the integral of (43). We have

$$
\left(\frac{\rho\left(r_{0}\right) c\left(r_{0}\right)^{3} r_{0}}{\rho(r) c(r)^{3} r}\right)^{1 / 2}=\frac{\rho(r)^{1 / 4}\left|r_{0}^{2}-r_{m}^{2}\right|^{3 / 2} r_{0}^{1 / 2}}{\rho\left(r_{0}\right)^{1 / 4}\left|r^{2}-r_{m}^{2}\right|^{3 / 2} r^{1 / 2}},
$$

Also

$$
\frac{2 \rho c^{2}+B^{2}+\rho^{2} P_{\rho \rho}}{2 \rho c^{2}}=\frac{3}{2}
$$

and

$$
\frac{B_{z} \rho\left(P_{\rho}+c^{2}\right)}{2 \rho^{2} c^{4}} \frac{\partial B_{r}}{\partial z}=-\frac{r^{2}}{r^{2}-r_{m}^{2}},
$$

so that

$$
\exp \left(\int_{r_{0}}^{r} \frac{B_{z} \rho\left(P_{\rho}+c^{2}\right)}{2 \rho^{2} c^{4}} \frac{\partial B_{r}}{\partial z} d s\right)=\exp \left(r_{0}-r\right)\left|\frac{r+r_{m}}{r-r_{m}}\right|^{r_{m} / 2}\left|\frac{r_{0}+r_{m}}{r_{0}-r_{m}}\right|^{-r_{m} / 2} .
$$

Equation (43) becomes now

$$
\begin{aligned}
& -\frac{1}{w\left(r_{0}\right)}=\frac{3}{8} e^{r_{0}} \rho\left(r_{0}\right)^{-1 / 4}\left|r_{0}-r_{m}\right|^{\left(3+r_{m}\right) / 2}\left|r_{0}+r_{m}\right|^{\left(3-r_{m}\right) / 2} \\
& \quad \times \int_{r_{0}}^{r_{1}} e^{-r} \rho(r)^{3 / 4}\left|r+r_{m}\right|^{\left(-5+r_{m}\right) / 2}\left|r-r_{m}\right|^{\left(-5-r_{m}\right) / 2} d r .
\end{aligned}
$$


Since due to the presence of the factor $\left|r-r_{m}\right|^{\left(-5-r_{m}\right) / 2}$ the integral is infinite as soon as the interval $\left[r_{0}, r_{1}\right]$, includes the magnetic axis $r_{m}$, for any initial condition $w\left(r_{0}\right)<0$ (if $r_{0}<r_{m}$ ) or $w\left(r_{0}\right)>0$ (if $r_{0}>r_{m}$ ) a shock wave forms before the wavefront reaches the magnetic axis. This is logical since the fact that the magnetic field vanishes there and the waves travel at the Alfvén velocity imply that no wave can cross the magnetic axis, so successive wavefronts pile there until a discontinuity is created.

\subsection{Accretion disks with non toroidal magnetic field}

Axisymmetric equilibria for accretion disks must take into account the presence of the gravitational term and the flow velocity; the result is a generalized GradShafranov equation where the pressure is non longer a function of the magnetic flux. It can be analytically solved if we assume that the temperature, the density or the entropy are functions of the flux. The results, when numerically integrated, show some differences between these three models [22]. In our case, however, we will only worry about the equilibrium equations at the plane $\Pi$, where many quantities vanish, and assume the existence of a general equilibrium coinciding with ours in the equator. Later we will even assume a self-similar form where all the quantities are powers of the radius $r$, and the toroidal velocity is Keplerian. The result cannot be valid for the whole length of an accretion disk, but we will restrict ourselves to the zone where this description holds, not too near to the central object.

Assuming the matter in an accretion disk flows towards the equator and rotates with the same direction in the northern and southern hemispheres, both reasonable assumptions, the velocity must satisfy that $v_{z}$ is odd, $v_{r}$ even and $v_{\phi}$ even. If the magnetic field starts with e.g. a dipole topology and then it is dragged by the flow, we must have $B_{z}$ even, $B_{r}$ odd and $B_{\phi}$ odd. The odd quantities vanish at $z=0$, whereas the derivatives with respect to $z$ of the even ones vanish there. Taking this into account, the equilibrium equations at $\Pi$ may 
be written as

$$
\begin{aligned}
-B_{z} \frac{\partial B_{r}}{\partial z}+B_{z} \frac{\partial B_{z}}{\partial r}+\rho v_{r} \frac{\partial v_{r}}{\partial r}+P_{\rho} \frac{\partial \rho}{\partial r}-\frac{\rho v_{\phi}^{2}}{r}+\frac{\rho G M}{r^{2}} & =0 \\
-B_{z} \frac{\partial B_{\phi}}{\partial z}+\rho v_{r} \frac{\partial v_{\phi}}{\partial r} & =0 \\
v_{r} \frac{\partial B_{z}}{\partial r}+B_{z} \frac{\partial v_{r}}{\partial r}+\frac{B_{z} v_{r}}{r} & =0 \\
\rho \frac{\partial v_{z}}{\partial z}+v_{r} \frac{\partial \rho}{\partial r}+\rho \frac{\partial v_{r}}{\partial r}+\frac{\rho v_{r}}{r} & =0 .
\end{aligned}
$$

From (63) one deduces $r v_{r} B_{z}=$ const. In all classical accretion disks one has $\left|v_{r}\right| \ll\left|v_{\phi}\right|$, and moreover the magnetic field decreases faster than $r^{-1}$, so we must take $v_{r}=0$ at $\Pi$. Taking this to (62) we find $\partial B_{\phi} / \partial z=0$, and to (64) $\partial v_{z} / \partial z=0$. We are left with $(61)$, which now reads

$$
B_{z}\left(\frac{\partial B_{z}}{\partial r}-\frac{\partial B_{r}}{\partial z}\right)+\frac{\partial P}{\partial r}-\frac{\rho v_{\phi}^{2}}{r}+\frac{\rho G M}{r^{2}}=0 .
$$

We assume that the velocity $v_{\phi}$ must be Keplerian, and the plasma polytropic, with $\gamma=5 / 3$. Taking a dependence of all magnitudes in powers of the radius, $[23]$,

$$
\rho=\rho_{0} r^{-3 / 2}, \quad P=P_{0} r^{-5 / 2}, \quad B_{z}=B_{z 0} r^{-5 / 4}, \quad v_{\phi}=v_{\phi 0} r^{-1 / 2},
$$

and taking this to (65), we find

$$
\frac{\partial B_{r}}{\partial z}=k r^{-9 / 4}
$$

with

$$
-B_{z 0}\left(\frac{5 B_{z 0}}{4}+k\right)-\frac{5}{2} P_{0}-\rho_{0} v_{\phi 0}^{2}+\rho_{0} G M=0 .
$$

Hence

$$
B_{z} \frac{\partial B_{r}}{\partial z}=\lambda r^{-7 / 2}=\left(-\frac{5}{4} B_{z 0}^{2}-\frac{5}{2} P_{0}-\rho_{0} v_{\phi 0}^{2}+\rho_{0} G M\right) r^{-7 / 2} .
$$

The expressions of $\mathbf{u}_{0}$ and its derivatives are shown in the appendix. Formula (43) remains valid, and its factors become

$$
\begin{array}{r}
\left(\frac{\rho_{0} c_{0}^{3} r_{0}}{\rho c^{3} r}\right)^{1 / 2}=\frac{r}{r_{0}} \\
1+\frac{B^{2}+\rho^{2} P_{\rho \rho}}{2 \rho c^{2}}=\frac{3 B_{z 0}^{2}+(40 / 9) P_{0}}{2 B_{z 0}^{2}+(10 / 3) P_{0}}=\kappa>0 \\
\frac{1}{c}=\left(\frac{\rho_{0}}{B_{z 0}^{2}+(5 / 3) P_{0}}\right)^{1 / 2}=\delta r^{-1 / 2} .
\end{array}
$$


The factor within the exponential is

$$
\frac{B^{2}+2 \gamma P}{2\left(\rho c^{2}\right)^{2}} B_{z} \frac{\partial B_{r}}{\partial z}=\frac{B_{z 0}^{2}+(10 / 3) P_{0}}{2\left(B_{z 0}^{2}+(5 / 3) P_{0}\right)^{2}} \lambda r^{-7 / 2} r^{5 / 2}=\mu r^{-1} .
$$

Notice that the sign of $\mu$ is the same as the one of $\lambda$. Hence its associated factor is

$$
\exp \left(\int_{r_{0}}^{r} \mu \frac{d s}{s}\right)=\left(\frac{r}{r_{0}}\right)^{\mu}
$$

so that (43) becomes

$$
-\frac{1}{w\left(r_{0}\right)}=\int_{r_{0}}^{r_{1}} \frac{\kappa \delta}{r_{0}^{\mu+1}} r^{\mu+1 / 2} d r=\frac{\kappa \delta}{r_{0}^{\mu+1}} \frac{1}{\mu+3 / 2}\left(r_{1}^{\mu+1 / 2}-r_{0}^{\mu+1 / 2}\right),
$$

for $\mu \neq-3 / 2$, and

$$
-\frac{1}{w\left(r_{0}\right)}=\kappa \delta r_{0}^{1 / 2} \ln \left(\frac{r_{1}}{r_{0}}\right)
$$

in the improbable case that $\mu=-3 / 2$. Notice that for $\mu+3 / 2>0$ and $r_{1}>r_{0}$, necessarily $w\left(r_{0}>0\right.$; the remaining possible combination of signs are equally easy. Always

$$
r_{1}=r_{0}\left(1-\frac{1}{w\left(r_{0}\right)} \frac{r_{0}^{1 / 2}(\mu+3 / 2)}{\kappa \delta}\right)^{1 /(\mu+1 / 2)},
$$

for $\mu \neq-3 / 2$, and

$$
r_{1}=r_{0} \exp \left(-\frac{1}{w\left(r_{0}\right) \kappa \delta r_{0}^{1 / 2}}\right)
$$

for $\mu=-3 / 2$. In theory this predicts precisely the point of formation of a shock wave. Obviously $r_{1}$ may be inaccessible because there the power description of the main quantities (66) does not hold.

\subsection{Cylindrical accretion rings}

If we wish to have a nontrivial toroidal field at the equator, we may take equilibria with $v_{r}=v_{z}=B_{r}=0, v_{\phi}, B_{z}, B_{\phi}$ even functions of $z$ [24]. The equilibrium equations reduce at the plane $\Pi$ to the single equation

$$
B_{z} \frac{\partial B_{z}}{\partial r}+B_{\phi} \frac{\partial B_{\phi}}{\partial r}+\frac{\partial P}{\partial r}-\frac{\rho v_{\phi}^{2}}{r}+\frac{B_{\phi}^{2}}{r}+\frac{\rho G M}{r^{2}}=0 .
$$


Positing a form of the quantities as powers of $r$, and a Keplerian velocity, we choose

$$
\begin{array}{r}
\rho=\rho_{0} r^{-3 / 2}, \quad P=P_{0} r^{-5 / 2}, \quad B_{z}=B_{z 0} r^{-5 / 4} \\
B_{\phi}=B_{\phi 0} r^{-5 / 4}, \quad v_{\phi}=v_{\phi 0} r^{-1 / 2}
\end{array}
$$

so that (77) reduces to

$$
-\frac{5}{4} B_{z 0}^{2}-\frac{1}{4} B_{\phi 0}^{2}-\frac{5}{2} P_{0}-\rho_{0} v_{\phi 0}^{2}+\rho_{0} G M=0 .
$$

Since $B_{r}=0$, the exponential term becomes 1 in equation (43). The remaining factors are

$$
\begin{array}{r}
\left(\frac{\rho_{0} c_{0}^{3} r_{0}}{\rho c^{3} r}\right)^{1 / 2}=\frac{r}{r_{0}} \\
1+\frac{B^{2}+\rho^{2} P_{\rho \rho}}{2 \rho c^{2}}=\frac{3 B_{z 0}^{2}+3 B_{\phi 0}^{2}+(40 / 9) P_{0}}{2 B_{z 0}^{2}+2 B_{\phi 0}^{2}+(10 / 3) P_{0}}=\kappa_{1}>0 \\
\frac{1}{c}=\left(\frac{\rho_{0}}{B_{z 0}^{2}+B_{\phi 0}^{2}+(5 / 3) P_{0}}\right)^{1 / 2}=\delta_{1} r^{-1 / 2} .
\end{array}
$$

Equation (43) is now

$$
-\frac{1}{w\left(r_{0}\right)}=\int_{r_{0}}^{r_{1}} r^{1 / 2} d r=\frac{2}{3} \frac{\kappa_{1} \delta_{1}}{r_{0}}\left(r_{1}^{3 / 2}-r_{0}^{3 / 2}\right) .
$$

Notice that for $r_{1}>r_{0}, w\left(r_{0}\right)<0$, and for $r_{1}<r_{0}, w\left(r_{0}\right)>0$. Thus

$$
r_{1}=\left(r_{0}^{3 / 2}-\frac{3 r_{0}}{2 \kappa_{1} \delta_{1} w(0)}\right)^{2 / 3}
$$

with the same caveats about the location of $r_{1}$ as before.

\section{Conclusions}

One rarely studied source of instability in axisymmetric equilibria is the possible formation of shock waves in the plasma. Since this is a nonlinear effect, it depends on an essential way on the size as well as the functional form of the initial perturbation. For the case where the solution of an hyperbolic nonlinear system moves into a stationary state, the equations describing the evolution as well as the possible degeneration of the wavefront into a shock are known. Since 
the quickest MHD wave as well as the one which may cross magnetic surfaces is the fast magnetosonic one, we study how this wave may start a shock into an axisymmetric equilibrium state. The result is given in the form of an integral identity relating the initial condition with the rest variables. This identity is analyzed in depth in three cases: static equilibria appropriate for the laboratory (without gravitational forcing) and described by the Grad-Shafranov equations, in particular in the case of low beta plasma; accretion rings with a nontrivial poloidal magnetic field, which are the most frequently posited, and cylindrical accretion rings. In these last two cases the main quantities are assumed to be function of a power of the radius, and the velocity to be Keplerian. In all cases a definite prediction on the location of the formation of the shock is made.

\section{Acknowledgment}

Partially supported by the Ministry of Economy and Innovation of Spain under contract MTM2012-31439.

\section{References}

[1] J.P. Freidberg, Ideal magnetohydrodynamic theory of magnetic fusion systems, Rev. of Mod. Phys. 54, 3 (1982) 801-902

[2] J.P. Goedbloed, R. Keppens, S. Poedts, Advanced Magnetohydrodynamics, Cambridge Univ. Press, Cambridge (2010)

[3] P.D. Lax, Hyperbolic systems of conservation laws II, Comm. Pure Appl. Math. 10 (1957) 537-566

[4] A. Jeffrey, T. Taniuti, Nonlinear Wave Propagation, Academic Press, NY (1964)

[5] E. Varley, E. Cumberbatch, Non-linear theory of wave-front propagation, J. Inst. Math. Appl. 1 (1965) 101-112 
[6] I. Bohachevsky, Simple waves and shocks in magnetohydrodynamics, Phys. Fluids 5 (1962) 1456-1467

[7] A. Jeffrey, The formation of magnetoacoustic shocks, J. Math. Anal. Appl. 11 (1965) 139-150

[8] A.G. Kulikovskiy, G.A. Lyubimov, Magneto-Hydrodynamics, AddisonWesley, Reading Mass. (1965)

[9] J. Frank, A. King, D. Raine, Accretion Power in Astrophysics (3rd edition), Cambridge Univ. Press, Cambridge, U.K. (2002)

[10] R.V.E. Lovelace, C. Mehanian, C.M. Mobarry, M.E. Sulkanen, Theory of axisymmetric magnetohydrodynamic flows: disks, Astrophys. J. Suppl. Series 62 (1986) 1-37

[11] R. Alexander, From disks to planetesimals I. evolution of gas and dust disks, New Astron. Rev. 52 (2008) 60-77

[12] G.P. Velikhov, Stability of an ideally conducting liquid flowing between cylinders rotating in a magnetic field, Sov. Phys. JETP 36 (1959) 995-998

[13] S.A. Balbus, F.H. Hawley, A powerful local shear instability in weakly magnetized disks I: linear analysis, Astrophys. J. 376 (1991) 214-222

[14] S.A. Balbus, F.H. Hawley, On the nature of angular momentum transport in nonradiative accretion flows, Astrophys. J. 573 (2002) 749-753

[15] H.C. Spruit, Shock waves in accretion disks, Rev. in Mod. Astron. 4 (1991) 197-207

[16] J.F. Heyvaerts, E.R. Priest, A model for a non-Keplerian magnetic accretion disk with a magnetically heated corona, Astron. Astrophys. 216 (1989) $230-244$

[17] G.B. Whitham, Linear and Nonlinear Waves, J. Wiley and Sons, NY (1974)

[18] T.Y. Thomas, Plastic Flow and Fracture in Solids, Academic Press, NY (1961) 
[19] P. Prasad, Nonlinear Hyperbolic Waves in Multi-Dimensions, Chapman and Hall/CRC Press, Boca Raton, FL (2001)

[20] V.D. Sharma, Quasilinear Hyperbolic Systems, Compressible Flows and Waves, CRC Press, Boca Raton, FL (2010)

[21] H. Cabannes, Theoretical Magnetofluid Dynamics, Academic Press, NY (1970)

[22] A.J.C. Beliën, M.A. Botchev, J.P. Goedbloed, B. van der Holst, R. Keppens. FINESSE: Axisymmetric MHD equilibria with flow. J. Comp. Phys., 182 (2002) 91-117

[23] H.C. Spruit, T. Matsuda, M. Inoue, K. Sawada, Spiral shocks and accretion in discs, Month. Not. Roy. Astr. Soc., 229 (1987, 517527

[24] F.H. Hawley, Global magnetohydrodynamic simulations of cylindrical keplerian disks, Astrophys. J. 554 (2001) 534547

\section{A Calculations for the equation coefficients}

1) The calculations of the left and right eigenvectors are routine. Denoting as asserted by $I_{7}$ the $7 \times 7$ identity matrix, the derivatives of the matrices $A_{r}$ and $A_{z}$ are as follows:

$$
\begin{gathered}
\nabla_{v_{z}} A_{r}=\nabla_{v_{\phi}} A_{r}=0, \quad \nabla_{v_{r}} A_{r}=I_{7} \\
\nabla_{B_{z}} A_{r}=\left[\begin{array}{ccccccc}
0 & 0 & 0 & 0 & 0 & 0 & 0 \\
0 & 0 & 0 & 1 / \rho & 0 & 0 & 0 \\
0 & 0 & 0 & 0 & 0 & 0 & 0 \\
0 & 1 & 0 & 0 & 0 & 0 & 0 \\
0 & 0 & 0 & 0 & 0 & 0 & 0 \\
0 & 0 & 0 & 0 & 0 & 0 & 0 \\
0 & 0 & 0 & 0 & 0 & 0 & 0
\end{array}\right]
\end{gathered}
$$




$$
\begin{aligned}
& \nabla_{B_{r}} A_{r}=\left[\begin{array}{ccccccc}
0 & 0 & 0 & -1 / \rho & 0 & 0 & 0 \\
0 & 0 & 0 & 0 & 0 & 0 & 0 \\
0 & 0 & 0 & 0 & 0 & -1 / \rho & 0 \\
-1 & 0 & 0 & 0 & 0 & 0 & 0 \\
0 & 0 & 0 & 0 & 0 & 0 & 0 \\
0 & 0 & -1 & 0 & 0 & 0 & 0 \\
0 & 0 & 0 & 0 & 0 & 0 & 0 \\
0 & 0 & 0 & 0 & 0 & 0 & 0
\end{array}\right] \\
& \nabla_{B_{\phi}} A_{r}=\left[\begin{array}{ccccccc}
0 & 0 & 0 & 0 & 0 & 0 & 0 \\
0 & 0 & 0 & 0 & 0 & 1 / \rho & 0 \\
0 & 0 & 0 & 0 & 0 & 0 & 0 \\
0 & 0 & 0 & 0 & 0 & 0 & 0 \\
0 & 0 & 0 & 0 & 0 & 0 & 0 \\
0 & 1 & 0 & 0 & 0 & 0 & 0 \\
0 & 0 & 0 & 0 & 0 & 0 & 0
\end{array}\right] \\
& \nabla_{\rho} A_{r}=\left[\begin{array}{ccccccc}
0 & 0 & 0 & B_{r} / \rho^{2} & 0 & 0 & 0 \\
0 & 0 & 0 & -B_{z} / \rho^{2} & 0 & -B_{\phi} / \rho^{2} & \partial / \partial \rho\left(P_{\rho} / \rho\right) \\
0 & 0 & 0 & 0 & 0 & B_{r} / \rho^{2} & 0 \\
0 & 0 & 0 & 0 & 0 & 0 & 0 \\
0 & 0 & 0 & 0 & 0 & 0 & 0 \\
0 & 0 & 0 & 0 & 0 & 0 & 0 \\
0 & 1 & 0 & 0 & 0 & 0 & 0
\end{array}\right] . \\
& \nabla_{v_{r}} A_{z}=\nabla_{v_{\phi}} A_{z}=0, \quad \nabla_{v_{z}} A_{z}=I_{7} \\
& \nabla_{B_{z}} A_{z}=\left[\begin{array}{ccccccc}
0 & 0 & 0 & 0 & 0 & 0 & 0 \\
0 & 0 & 0 & 0 & -1 / \rho & 0 & 0 \\
0 & 0 & 0 & 0 & 0 & -1 / \rho & 0 \\
0 & 0 & 0 & 0 & 0 & 0 & 0 \\
0 & -1 & 0 & 0 & 0 & 0 & 0 \\
0 & 0 & -1 & 0 & 0 & 0 & 0 \\
0 & 0 & 0 & 0 & 0 & 0 & 0
\end{array}\right]
\end{aligned}
$$




$$
\begin{aligned}
& \nabla_{B_{r}} A_{z}=\left[\begin{array}{ccccccc}
0 & 0 & 0 & 0 & 1 / \rho & 0 & 0 \\
0 & 0 & 0 & 0 & 0 & 0 & 0 \\
0 & 0 & 0 & 0 & 0 & 0 & 0 \\
0 & 0 & 0 & 0 & 0 & 0 & 0 \\
1 & 0 & 0 & 0 & 0 & 0 & 0 \\
0 & 0 & 0 & 0 & 0 & 0 & 0 \\
0 & 0 & 0 & 0 & 0 & 0 & 0
\end{array}\right] \\
& \nabla_{B_{\phi}} A_{z}=\left[\begin{array}{ccccccc}
0 & 0 & 0 & 0 & 0 & 1 / \rho & 0 \\
0 & 0 & 0 & 0 & 0 & 0 & 0 \\
0 & 0 & 0 & 0 & 0 & 0 & 0 \\
0 & 0 & 0 & 0 & 0 & 0 & 0 \\
0 & 0 & 0 & 0 & 0 & 0 & 0 \\
1 & 0 & 0 & 0 & 0 & 0 & 0 \\
0 & 0 & 0 & 0 & 0 & 0 & 0
\end{array}\right] \\
& \nabla_{\rho} A_{z}=\left[\begin{array}{ccccccc}
0 & 0 & 0 & 0 & -B_{r} / \rho^{2} & -B_{\phi} / \rho^{2} & \partial / \partial \rho\left(P_{\rho} / \rho\right) \\
0 & 0 & 0 & B_{z} / \rho^{2} & 0 & 0 & 0 \\
0 & 0 & 0 & 0 & 0 & B_{z} / \rho^{2} & 0 \\
0 & 0 & 0 & 0 & 0 & 0 & 0 \\
0 & 0 & 0 & 0 & 0 & 0 & 0 \\
0 & 0 & 0 & 0 & 0 & 0 & 0 \\
1 & 0 & 0 & 0 & 0 & 0 & 0
\end{array}\right] .
\end{aligned}
$$

In all the cases under study we have $B_{r}=0$ at the plane $\Pi$. Then

$$
\begin{array}{r}
\mathbf{R} \cdot \nabla_{u} A_{r}=c I_{7}+B_{z} \nabla_{B_{z}} A_{r}+B_{\phi} \nabla_{B_{\phi}} A_{r}+\rho \nabla_{\rho} A_{r} \\
=c I_{7}+\left[\begin{array}{ccccccc}
0 & 0 & 0 & 0 & 0 & 0 & 0 \\
0 & 0 & 0 & 0 & 0 & 0 & \rho \partial / \partial \rho\left(P_{\rho} / \rho\right) \\
0 & 0 & 0 & 0 & 0 & 0 & 0 \\
0 & B_{z} & 0 & 0 & 0 & 0 & 0 \\
0 & 0 & 0 & 0 & 0 & 0 & 0 \\
0 & B_{\phi} & 0 & 0 & 0 & 0 & 0 \\
0 & \rho & 0 & 0 & 0 & 0 & 0
\end{array}\right] .
\end{array}
$$


Thus

$$
\left(\nabla_{u} A_{r} \cdot \mathbf{R}\right) \cdot \mathbf{R}=c \mathbf{R}+\left(0, \rho^{2} \frac{\partial}{\partial \rho}\left(\frac{P_{\rho}}{\rho}\right), 0, c B_{z}, 0, c B_{\phi}, c \rho\right)
$$

and

$$
\begin{aligned}
q_{0} & =\frac{1}{c} \mathbf{L} \cdot\left(\nabla_{u} A_{r} \cdot \mathbf{R}\right) \cdot \mathbf{R} \\
=2 \rho c^{2}+\rho^{3} \frac{\partial}{\partial \rho}\left(\frac{P_{\rho}}{\rho}\right)+B_{z}^{2}+B_{\phi}^{2}+\rho P_{\rho} & =2 \rho c^{2}+B^{2}+\rho^{2} P_{\rho \rho} .
\end{aligned}
$$

Since for all cases

$$
\frac{\partial \mathbf{R}}{\partial r}=\left(0, \frac{\partial c}{\partial r}, 0, \frac{\partial B_{z}}{\partial r}, 0, \frac{\partial B_{\phi}}{\partial r}, \frac{\partial \rho}{\partial r}\right)
$$

formula (29) is routine.

To obtain (30) we need to find

$$
\nabla_{u} \mathbf{C} \cdot \mathbf{R}=c \frac{\partial \mathbf{C}}{\partial v_{r}}+B_{z} \frac{\partial \mathbf{C}}{\partial B_{z}}+B_{\phi} \frac{\partial \mathbf{C}}{\partial B_{\phi}}+\rho \frac{\partial \mathbf{C}}{\partial \rho} .
$$

We have

$$
\begin{aligned}
\frac{\partial \mathbf{C}}{\partial v_{r}} & =\left(0,0, \frac{v_{\phi}}{r}, \frac{B_{z}}{r}, \frac{B_{r}}{r}, 0, \frac{\rho}{r}\right) \\
\frac{\partial \mathbf{C}}{\partial B_{z}} & =\left(0,0,0, \frac{v_{r}}{r}, 0,0,0\right) \\
\frac{\partial \mathbf{C}}{\partial B_{\phi}} & =\left(0, \frac{2 B_{\phi}}{\rho r},-\frac{B_{r}}{\rho r}, 0,0,0,0\right) \\
\frac{\partial \mathbf{C}}{\partial \rho} & =\left(0,-\frac{B_{\phi}^{2}}{\rho r}, \frac{B_{r} B_{\phi}}{\rho^{2} r}, 0,0,0, \frac{v_{r}}{r}\right) .
\end{aligned}
$$

Thus, for $B_{r}=0$

$$
\begin{array}{r}
\nabla_{u} \mathbf{C} \cdot \mathbf{R}=\left(0, \frac{B_{\phi}^{2}}{\rho r}, 0, \frac{c B_{z}}{r}, 0,0, \frac{c \rho}{r}\right) \\
\mathbf{L} \cdot\left(\nabla_{u} \mathbf{C} \cdot \mathbf{R}\right)=\rho c \frac{B_{\phi}^{2}}{\rho r}+\frac{c B_{z}^{2}}{r}+\frac{c \rho P_{\rho}}{r}=\frac{\rho c^{3}}{r} .
\end{array}
$$

The equilibrium states have the form at $\Pi$

$$
\begin{array}{ll}
\mathbf{u}_{0}=\left(0,0,0, B_{z}, 0, B_{\phi}, \rho\right) & (\text { Case } 4.1) \\
\mathbf{u}_{0}=\left(0, v_{r}, v_{\phi}, B_{z}, 0,0, \rho\right) & (\text { Case } 4.2) \\
\mathbf{u}_{0}=\left(0,0, v_{\phi}, B_{z}, 0, B_{\phi}, \rho\right) & (\text { Case 4.3) }
\end{array}
$$


and satisfy

$$
\begin{array}{rlr}
\frac{\partial \mathbf{u}_{0}}{\partial r} & =\left(0,0,0, \frac{\partial B_{z}}{\partial r}, 0, \frac{\partial B_{\phi}}{\partial r}, \frac{\partial \rho}{\partial r}\right) & (\text { Case 4.1) } \\
\frac{\partial \mathbf{u}_{0}}{\partial r} & =\left(0,0, \frac{\partial v_{\phi}}{\partial r}, \frac{\partial B_{z}}{\partial r}, 0,0, \frac{\partial \rho}{\partial r}\right) & (\text { Case 4.2) } \\
\frac{\partial \mathbf{u}_{0}}{\partial r} & =\left(0,0, \frac{\partial v_{\phi}}{\partial r}, \frac{\partial B_{z}}{\partial r}, 0, \frac{\partial B_{\phi}}{\partial r}, \frac{\partial \rho}{\partial r}\right) & (\text { Case 4.3) }
\end{array}
$$

as well as

$$
\begin{aligned}
\frac{\partial \mathbf{u}_{0}}{\partial z} & =\left(0,0,0,0, \frac{\partial B_{r}}{\partial z}, 0,0\right) & & (\text { Case 4.1) } \\
\frac{\partial \mathbf{u}_{0}}{\partial z} & =\left(0,0,0,0, \frac{\partial B_{r}}{\partial z}, 0,0\right) & & (\text { Case 4.2) } \\
\frac{\partial \mathbf{u}_{0}}{\partial z} & =\mathbf{0} \quad(\text { Case } 4.3) . & &
\end{aligned}
$$

The expression of $\partial \mathbf{R} / \partial r$ is already known (96). As for $\partial \mathbf{R} / \partial z$, we have the general form

$$
\frac{\partial \mathbf{R}}{\partial z}=\left(-\frac{B_{z}}{\rho c}, 0,-\frac{B_{\phi}}{\rho c}, 0,0,0,0\right) \frac{\partial B_{r}}{\partial z},
$$

which degenerates into

$$
\begin{array}{ll}
\frac{\partial \mathbf{R}}{\partial z} & =\left(-\frac{B_{z}}{\rho c}, 0,0,0,0,0,0\right) \frac{\partial B_{r}}{\partial z} \quad(\text { Case 4.2) } \\
\frac{\partial \mathbf{R}}{\partial z} & =\mathbf{0} \quad(\text { Case } 4.3) .
\end{array}
$$

Since we have all the needed vectors and matrices, the proof of (34-36) is now a mere calculation. 